\title{
Frontal Plane Biomechanics during Walking
}

\author{
Anne Schmitz ${ }^{1 *}$ and Jaclyn Norberg ${ }^{2}$ \\ ${ }^{1}$ Department of Mechanical Engineering, Gannon University, USA \\ ${ }^{2}$ Department of Sport and Movement Science, Salem State University, USA
}

Submission: January 22, 2018; Published: February 07, 2018

*Corresponding author: Anne Schmitz, Department of Mechanical Engineering, Gannon University, 109 University Square, Erie, PA 16541, Tel: (814) 871-5799; Email: schmitz005@gannon.edu

\begin{abstract}
The goal of this study was to test the assumption that the ground reaction force (GRF) passes through the whole body's center of mass (COM) in the frontal plane during walking. Whole body kinematics were analyzed for 17 subjects $(7$ male, 10 female, age $24 \pm 4$ years old, height $1.71 \pm 0.07 \mathrm{~m}$, mass $66.1 \pm 12.4 \mathrm{~kg})$ walking at a self-selected speed $(1.17 \pm 0.10 \mathrm{~m} / \mathrm{s})$ on an instrumented treadmill. The time rate of change of the angular momentum of the body in the frontal plane with respect to the lab coordinate system was calculated, along with the magnitude of the GRF in the frontal plane, and the moment arm of the GRF about the body's COM. The moment arm of the GRF about the COM in the frontal plane ranged from -33 to $27 \mathrm{~mm}$ during gait. This is contrary to what has been assumed previously in the literature where the GRF has been presumed to pass through the body's COM when describing the influence of trunk motion on lower limb joint loading. Our results suggest the relation between trunk motion and knee joint loading may require an alternative explanation than is currently presented in the literature.
\end{abstract}

Keywords: Whole body center of mass; Frontal plane, Gait, Ground reaction force

\section{Introduction}

An untested assumption used in the literature is that the ground reaction force (GRF) passes through the body's center of mass ( $\mathrm{COM})$ in the frontal plane. Specifically, this has been used to describe the influence of trunk motion on lower limb joint loading in the frontal plane $[1,2]$. As the trunk leans towards the stance limb during gait, the GRF is thought to alter its line of action to pass through the COM, decreasing the moment arm of the GRF about the knee joint, and thereby decreasing the knee adduction moment [1]. Since the knee adduction moment has been used as a surrogate measure for knee joint loads, this relationship between trunk lean, GRF direction, and COM has been proposed as a mechanism to decrease loading of the knee joint [3]. However, there is no experimental evidence to support the trunk lean mechanism: that the GRF passes through the COM. Therefore, the goal of this study was to determine if the GRF passes through the COM in the frontal plane during walking.

\section{Methods}

\section{Data collection}

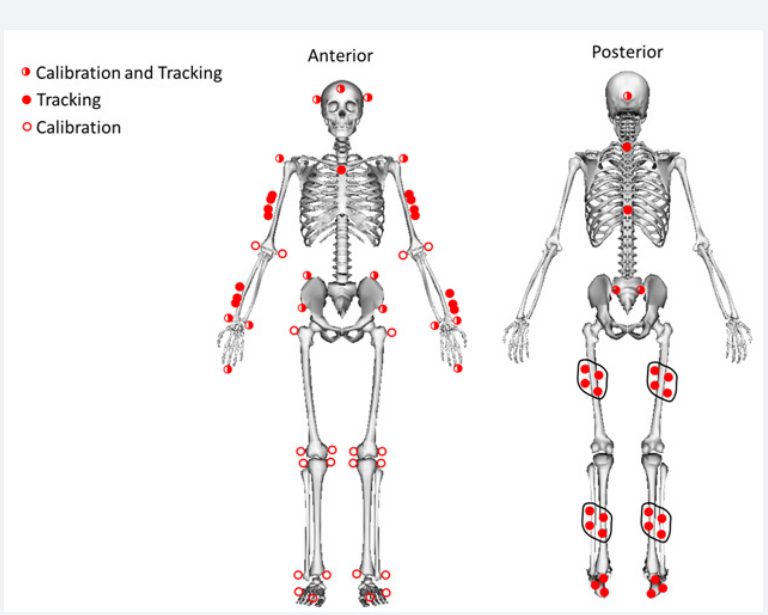

Figure 1: 79 markers were placed on the body: 40 anatomical markers and 39 additional tracking markers. 
This study is a secondary analysis of previously collected data [4] on 17 subjects ( 7 male, 10 female, age $24 \pm 4$ years old, height $1.71 \pm 0.07 \mathrm{~m}$, mass $66.1 \pm 12.4 \mathrm{~kg}$ ) walking at a selfselected speed $(1.17 \pm 0.10 \mathrm{~m} / \mathrm{s})$ on a treadmill. Instrumented gait analysis data was collected for the whole body by placing retroreflective markers on the lower [5] and upper [6] body (Figure 1). To minimize any differences due to shoe design, all subjects wore New Balance WR662 shoes (New Balance, Brighton, MA, USA).

Prior to data collection, the subjects walked at a self-selected pace for five minutes to acclimatize to the treadmill. Then, threedimensional marker trajectories were captured at $200 \mathrm{~Hz}$ using a ten-camera motion analysis system (Motion Analysis Corp, Santa Rosa, USA) while each subject walked for three minutes on an instrumented Bertec treadmill (Bertec Corp, Columbus, Ohio) collecting force plate data at $1000 \mathrm{~Hz}$. Data was only collected at the last 20 seconds of the third minute.

\section{Data analysis}

Visual 3D (C-motion, Germantown, MD, USA) was used to first low pass filter the marker and force plate data at $8 \mathrm{~Hz}$ and 35 $\mathrm{Hz}$, respectively, using a fourth order, zero-lag Butterworth filter. Second, the angular momentum of the whole body in the frontal plane with respect to the lab coordinate system was extracted where the mass properties of each segment were modeled as frustums [7]. Each joint was modeled as a 6 degree-of-freedom joint with segment fixed coordinate systems described in the supplemental information. Third, the magnitude of the GRF in each direction was also obtained. Matlab (MathWorks Inc., Natick, MA) was then used to calculate the time rate of change of the angular momentum, the magnitude of the GRF in the frontal plane, and the moment arm of the GRF about the body's COM. The moment arm was calculated according to the free body diagram and equations of motion in Figure 2.
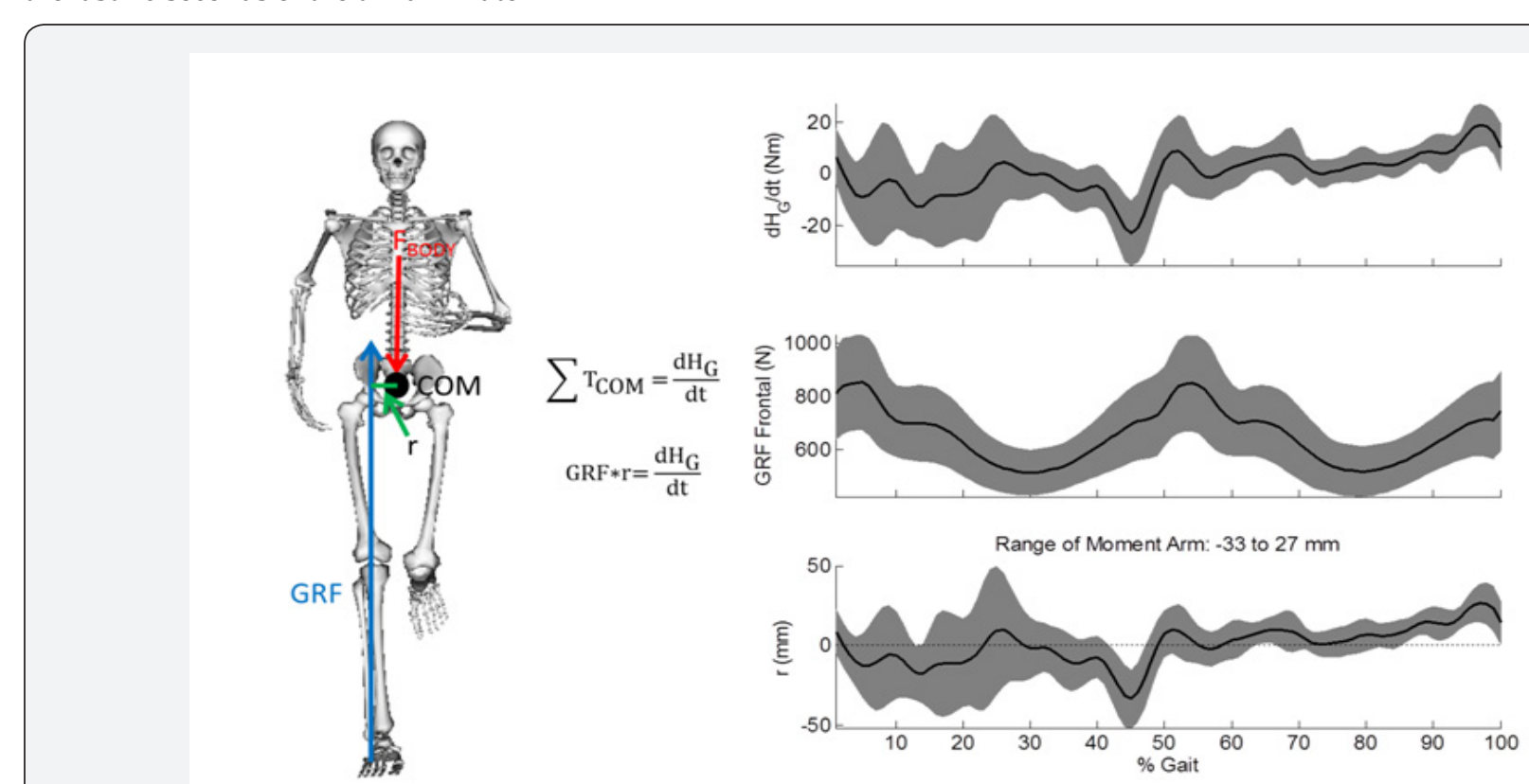

Figure 2: (left) Free body diagram and rotational equations of motion of the whole body during gait used to calculate the moment arm of the GRF about the COM. $F_{\text {BODY }}=$ weight of the body. COM= whole body center of mass. $r=$ moment arm of the GRF. GRF = ground reaction force. $\mathrm{T}_{\text {com }}=$ torque about the center of mass. $\mathrm{dH}_{\mathrm{G}} / \mathrm{dt}=$ time rate of change of angular momentum. (right) Ensemble curves (mean \pm standard deviation) comprised of 17 subjects, 1 trial per subject, of the time rate of change of angular momentum, GRF, and $r$ during gait. Notice how the moment arm varies throughout the gait cycle, ranging from -33 to $27 \mathrm{~mm}$ and averaging $0.3 \mathrm{~mm}$ across the cycle.

\section{Results}

The moment arm of the GRF about the COM in the frontal plane ranged from -33 to $27 \mathrm{~mm}$ and averaged $0.3 \mathrm{~mm}$ throughout the gait cycle (Figure 2).

\section{Discussion}

The moment arm of the GRF about the COM in the frontal plane varies during walking and cannot be assumed to pass through the COM (Figure 1). This is contrary to what has been stated previously in the literature [1,2], especially when describing the mechanism behind trunk sway and knee joint loading. Increased trunk sway has been associated with a decreased knee adduction moment [1]. The theory is that trunk motion shifts the body's COM location, altering the line of action of the GRF so it passes through the COM, modifying its moment arm about the knee, and effectively altering the knee adduction moment [1]. However, the results of the current study contradict the assumption used in this theory and suggest this mechanism may need to be refined. In agreement with our results, previous work has shown no correlation between the trunk's COM location and GRF magnitude in either direction [8]. An alternative mechanism that could be further explored is how trunk lean alters the location of the center of pressure of the GRF (COP) since the trunk's COM has been shown to be significantly correlated with COP [8]. Another alternative explanation could 
be gleaned from results looking at the sagittal plane. In the sagittal plane, the GRF passes through a point superior to the COM rather than through the COM to maintain stability during walking [9]. This could be further explored to see if this extends to the frontal plane to better explain the relation between trunk sway and the knee adduction moment.

There are some limitations and study design factors to consider. First, the subjects walked on a treadmill as opposed to over ground. Temporal gait parameters, kinematic patterns, and ground reaction force patterns have been found to be similar between treadmill and over ground walking [10-13]. Therefore, we expect our results to be applicable to over ground walking. Second, since only walking was analyzed, these results cannot be generalized to other movements such as running or jumping.

\section{Conclusion}

In conclusion, the GRF did not pass through the COM in the frontal plane during walking which contradicts what is assumed Supplemental information: in the literature when explaining the relation between trunk sway and the knee adduction moment. Our results suggest an alternative explanation may need to be further developed to understand this relation.

\section{Data Availability}

The motion capture data of this study is not publically available. Access to the data will be considered by the author upon request. For inquiries about data access, contact: Jaclyn Norberg, 352 Lafayette Street, Salem, MA 01970, Tel: (978) 5422779; Email: jaclyn.norberg@salemstate.edu

\section{Conflicts of Interest}

There are no sources to disclose.

\section{Acknowledgement}

Partial funding provided by NSF IIS 1231545. (Supplemental information)

\section{Supplemental Information Describing the Segment Fixed Coordinate Systems}

The segment fixed coordinate systems were created in software Visual 3D (C-motion, Germantown, MD, USA) using the following conventions:

\section{Foot}

Modified Oxford foot model as explained in [11].

\section{Shank}

Origin: Midpoint of line connecting the lateral femoral epicondyle and the medial tibial plateau

Z-axis: Connects origin with midpoint of offset malleoli markers

XZ-plane: Least-squares plane fit through the lateral tibial plateau, origin, and the offset malleoli markers

\section{Thigh}

Origin: Derived using the regression equations of $[12,13]$.

Z-axis: Connects origin with midpoint of lateral and medial femoral epicondyle

XZ-plane: Least-squares plane fit through the greater trochanter, origin, and lateral and medial femoral epicondyle

\section{Thorax/Ab (Contained Mass of Head)}

Origin: Midpoint of the iliac crests

Z-axis: Connects origin and midpoint of left and right acromion processes

XZ-plane: Least-squares plane fit through the iliac crests and acromion processes

\section{Head (Kinematic Segment)}

Origin: Midpoint of the lateral and medial aspects of the head

Z-axis: Connects origin and the top of the head

XZ-plane: Least-squares plane fit through top, medial, and lateral aspects of the head

\section{Upper Arm}

Origin: Acromion process

Z-axis: Connects origin with midpoint of lateral and medial epicondyles 
XZ-plane: Least-squares plane fit through a point projected $-0.05 \mathrm{~m}$ superiorly from the acromion process, origin, lateral epicondyle, and medial epicondyle

\section{Forearm}

Origin: At a point $0.04 \mathrm{~m}$ offset from the lateral epicondyle on a line connecting the medial and lateral epicondyles

Z-axis: Connects origin and midpoint of radial and ulnar styloid process

\section{References}

1. Hunt MA, Birmingham TB, Bryant D, Jones I, Giffin JR. et al. (2008) Lateral trunk lean explains variation in dynamic knee joint load in patients with medial compartment knee osteoarthritis. Osteoarthritis Cartilage 16(5): 591-599.

2. Powers CM (2010) The influence of abnormal hip mechanics on knee injury: a biomechanical perspective. J Orthop Sports Phys Ther 40(2): 42-51.

3. Fregly BJ (2012) Gait Modification to Treat Knee Osteoarthritis. HSS 8(1): 45-48.

4. IJmker T, Lamoth CJ, Houdijk H, Tolsma M, van der Woude LH, et al. (2013) The Effect of Handrail Holding on Treadmill Walking Kinematics. in American College of Sports Medicine Annual Meeting.

5. Noehren B, Pohl MB, Sanchez Z, Cunningham T, Lattermann C (2012) Proximal and distal kinematics in female runners with patellofemoral pain. Clin Biomech 27(4) ): 366-371.

6. Shapiro R (2012) Differences in Running and Walking Gait Kinematics during Earth and Simulated Mars and Lunar Gravitational Environments: Preliminary Investigation. In Americal Society of Biomechanics.
7. Hanavan EP (1964) A mathematical model of the human body. pp. 1-149.

8. Schmitz A, Noehren B (2014) What predicts the first peak of the knee adduction moment? Knee 21(6): 1077-1083.

9. Gruben KG, Boehm WL (2012) Force direction pattern stabilizes sagittal plane mechanics of human walking. Human Move Sci 31(3): 649-659.

10. Lee SJ, Hidler J (2008) Biomechanics of overground vs. treadmill walking in healthy individuals. J Appl Phys 104(3): 747-755.

11. Pohl MB, Messenger N, Buckley JG (2007) Forefoot, rearfoot and shank coupling: effect of variations in speed and mode of gait. Gait \& Posture 25(2): 295-302.

12. Bell AL, Brand RA, Pedersen DR (1989) Prediction of hip joint centre location from external landmarks. Human Move Sci8(1): 3-16.

13. Bell AL, Pedersen DR, Brand RA (1990) A comparison of the accuracy of several hip center location prediction methods. J Biomech 23(6): 617-621.

\begin{tabular}{l} 
Your next submission with Juniper Publishers \\
will reach you the below assets \\
- Quality Editorial service \\
- Swift Peer Review \\
- Reprints availability \\
- E-prints Service \\
- Manuscript Podcast for convenient understanding \\
- Global attainment for your research \\
- Manuscript accessibility in different formats \\
( Pdf, E-pub, Full Text, Audio) \\
- Unceasing customer service \\
Track the below URL for one-step submission \\
https://juniperpublishers.com/online-submission.php \\
\hline
\end{tabular}

